\section{A zebrafish model for HAX1-associated congenital neutropenia}

\author{
Larissa Doll, ${ }^{1}$ Narges Aghaallaei, ${ }^{1}$ Advaita M. Dick, ${ }^{1}$ Karl Welte, ${ }^{2}$ \\ Julia Skokowa ${ }^{1}$ and Baubak Bajoghli ${ }^{1}$ \\ ${ }^{1}$ Department of Oncology, Hematology, Immunology and Rheumatology, University \\ Hospital, University of Tübingen and 'University Children's Hospital Tübingen, Tübingen, \\ Germany
}

\section{ABSTRACT}

S evere congenital neutropenia is a rare heterogeneous group of diseases, characterized by an arrest of granulocyte maturation. Autosomal recessive mutations in the HAX1 gene are frequently detected in affected individuals. However, the precise role of HAX1 during neutrophil differentiation is poorly understood. To date, no reliable animal model has been established to study HAX1-associated congenital neutropenia. Here we show that knockdown of zebrafish hax1 impairs neutrophil development without affecting other myeloid cells and erythrocytes. Furthermore, we found that interference with Hax1 function decreases the expression level of key target genes of the granulocyte colony-stimulating factor signaling pathway. The reduced neutrophil numbers in the morphants could be reversed by granulocyte colony-stimulating factor, which is also the main therapeutic intervention for patients who have congenital neutropenia. Our results demonstrate that the zebrafish is a suitable model for HAX1-associated neutropenia. We anticipate that this model will serve as an in vivo platform to identify new avenues for developing tailored therapeutic strategies for patients with congenital neutropenia, particularly for those individuals who do not respond to granulocyte colony-stimulating factor treatment.

\section{Introduction}

Severe congenital neutropenia $(\mathrm{CN})$ is a rare hematologic disorder, which is characterized by impaired maturation of neutrophil granulocytes. ${ }^{1-6}$ Mutations in various genes, including ELANE, HAX1, G6PC3, CXCR4 and G-CSFR are associated with $\mathrm{CN} .{ }^{1}$ Affected individuals are prone to life-threatening infections that begin in their first months of life unless treated by human granulocyte colony-stimulating factor (G-CSF, encoded by the CSF3 gene) or bone marrow transplantation. ${ }^{1,2} \mathrm{CN}$ is also a preleukemic syndrome and patients are predisposed to develop myelodysplasia or acute myeloid leukemia after a long period of time ${ }^{7,8}$ However, the severity of neutropenia and the risk of leukemia vary between individuals with distinct genetic aberrations, and a group of CN patients do not respond to the G-CSF therapy. The cumulative incidence of leukemia in less responsive patients is $40 \%$ after 15 years, in comparison to $20 \%$ in responsive patients, ${ }^{9}$ although, the underlying mechanisms are poorly understood.

HAX1 is ubiquitously expressed among human tissues. ${ }^{10,11}$ It acts as a binding partner of multiple proteins and is involved in various signaling pathways and cellular processes. ${ }^{12-14}$ For example, HAX1 interacts with the mitochondrial proteases PARL and HTRA2 and is involved in anti-apoptotic signaling. ${ }^{15}$ It interacts with $\mathrm{PKD} 2$ protein and is associated with the actin cytoskeleton. ${ }^{16} \mathrm{HAX} 1$ plays a role in BCR-mediated internalization through binding to the cytoplasmic domain of B-cell receptors. ${ }^{17}$ In hematopoietic progenitors, upon activation of the G-CSF receptor (G-CSFR), the cytoplasmic HAX1 binds to the HCSL1 and LEF-1 proteins, transporting LEF-1 into the nucleus. This transcription factor then activates target genes of the G-CSF signaling pathway including CEBPA and HCLS $1 .^{18}$ Taking into account that HAX1 is involved in diverse subcellular processes, it remains unclear why patients who lack functional HAX1 display predominantly impaired neutrophil differentiation.

Our current knowledge on the role of HAX1 in granulopoiesis is predominantly derived from in vitro studies and clinical observations. ${ }^{1,2}$ To date, no faithful animal
Haematologica 2021

Volume 106(5):1311-1320

\section{Correspondence:}

BAUBAK BAJOGHLI

baubak.bajoghli@med.uni-tuebingen.de

Received: October 11, 2019.

Accepted: April 9, 2020.

Pre-published: April 23, 2020.

https://doi.org/10.3324/haematol.2019.240200

(C2021 Ferrata Storti Foundation

Material published in Haematologica is covered by copyright. All rights are reserved to the Ferrata Storti Foundation. Use of published material is allowed under the following terms and conditions:

https://creativecommons.org/licenses/by-nc/4.0/legalcode. Copies of published material are allowed for personal or internal use. Sharing published material for non-commercial purposes is subject to the following conditions:

https://creativecommons.org/licenses/by-nc/4.0/legalcode, sect. 3. Reproducing and sharing published material for commercial purposes is not allowed without permission in writing from the publisher. 
model has been established and, therefore, the experimental model system is based on in vitro neutrophil differentiation of induced pluripotent stem cells from patients with $H A X 1$-associated CN. ${ }^{19,20}$ Mice lacking Hax1 display normal granulopoiesis and their predominant phenotype is post-natal lethality due to neuronal apoptosis. ${ }^{15}$ Hence, there is an urgent need for an in vivo model to study the role of HAX1 in granulopoiesis and leukemogenesis. Here, we chose to use zebrafish (Danio rerio) as a vertebrate model because cellular and molecular mechanisms underlying granulopoiesis are largely conserved between zebrafish and humans, ${ }^{21-25}$ and $82 \%$ of diseasecausing human genes have an orthologue in zebrafish. ${ }^{26,27}$ For example, zebrafish harboring mutations in g-csfr, ${ }^{23,24}$ $\mathrm{cxcr} 4{ }^{28}$ and $\mathrm{g} 6 \mathrm{pc} 3^{29}$ display impaired neutrophil development, mimicking $\mathrm{CN}$ patients who lack the corresponding orthologue gene. Thus far, it was unknown whether hax1 is involved in zebrafish granulopoiesis. Here, we show that this gene is expressed in zebrafish hematopoietic cells and is indispensable for neutrophil development, which makes zebrafish a suitable model for studying $H A X 1$-associated neutropenia.

\section{Methods}

\section{Zebrafish}

Zebrafish lines were maintained according to standard protocols and handled in accordance with European Union animal protection directive 2010/63/EU and approved by the local government (Tierschutzgesetz $₫ 11$, Abs. 1 , Nr. 1, husbandry permit 35/9185.46/Uni TÜ). All experiments described in the present study were conducted on embryos younger than 5 days post-fertilization (dpf). In this study, we used the wild-type TE strain of zebrafish. The transgenic ( $\operatorname{tg})$ reporter lines $\operatorname{tg}(m p o: g f p)$ and $\operatorname{tg}(l y z: d s R E D)$ have been described previously. ${ }^{30,31}$

\section{Injection of morpholino, single-guide RNA and messenger RNA}

Three morpholinos (GeneTools) targeting the hax1 gene were used in this study (Online Supplementary Table S1). For the rescue experiment, full-length complementary DNA (cDNA) of zebrafish hax1 was isolated and cloned into the pMC vector. ${ }^{32}$ Capped messenger RNA (mRNA) was synthesized using the mMESSAGE mMACHINE SP6 kit (Ambion). Morpholino and 5$10 \mathrm{ng} / \mu \mathrm{L}$ of hax1 mRNA were co-injected into one-cell stage zebrafish embryos. To perform transient CRISPR-Cas9 targeting of the hax1 gene, single guide RNA (sgRNA) target site (5'GGGTTTTTCGGGATTCCCGG-3') was predicted and evaluated for off-target site by using the CCTop web tool. ${ }^{33}$ sgRNA (15 $\mathrm{ng} / \mu \mathrm{L}$ ) and $150 \mathrm{ng} / \mu \mathrm{L}$ of Cas9 mRNA (a kind gift from J. Wittbrodt, Heidelberg University) were co-injected into one-cell stage transgenic tg(mpo:gfp) embryos.

\section{Heat-inducible g-csfa construct}

The full-length cDNA of zebrafish g-csfa (or $c s f 3 a$ ) was isolated and cloned into the pTGH2 plasmid containing a bi-directional heat-inducible promoter ${ }^{34}$ flanked by Tol2 binding sites. The resulting plasmids were then injected at a dose of $20 \mathrm{ng} / \mu \mathrm{L}$ with $10 \mathrm{ng} / \mu \mathrm{L}$ mRNA of Tol2 transposase into one-cell-stage embryos. Injected embryos at $1 \mathrm{dpf}$ were heat treated at $39^{\circ} \mathrm{C}$ for $1 \mathrm{~h}$. Green fluorescent protein-positive $\left(\mathrm{GFP}^{+}\right)$embryos were selected for subsequent whole mount in situ hybridization (WISH) analysis.

\section{Whole mount in situ hybridization}

RNA in situ hybridization of zebrafish embryos was performed as described previously ${ }^{35}$ using digoxigenin-labeled RNA antisense probes, which are listed in Online Supplementary Table S2.

\section{Statistical analysis}

GraphPad Prism software (version 8) was used to produce graphs and for the statistical analysis. In this study, an unpaired, two-tailed Wilcoxon-Mann-Whitney test was used to compare the means of different data sets.

Additional details of the material and methods are available in the Online Supplementary Material and Methods.

\section{Results}

\section{Zebrafish hax1 is expressed in hematopoietic cells}

An in silico analysis was carried out to characterize zebrafish hax1. A high degree of synteny was found between human and zebrafish hax 1 with the upab2l and pygo 2 genes upstream, and $a q p 10 b$ and cks $1 b$ genes downstream in the corresponding genomic loci (Figure 1A). Multiple alignment and phylogenetic analysis revealed strong amino acid similarities over the entire coding region of Hax1 protein between zebrafish and its orthologues in other vertebrates (Figure 1B, Online Supplementary Figure S1). To elucidate whether hax1 is expressed in zebrafish hematopoietic cells, we first searched for hax 1 in two single-cell RNA-sequencing databases $^{36,37}$ of adult zebrafish whole kidney marrow (akin to mammalian bone marrow). In both databases, hax 1 was found to be expressed in neutrophils, macrophages, and erythrocytes, albeit at low levels (data not shown). WISH was performed to determine the hax 1 spatial expression patterns during embryonic development, revealing that hax1 is ubiquitously expressed throughout gastrulation and early embryonic segmentation (Figure 1C). At later stages, the hax1 transcript was detected in the posterior intermediate cell mass and caudal hematopoietic tissue (Figure 1C-F, black arrows), which are the sites of primitive and definitive hematopoiesis, respectively, in zebrafish embryos. ${ }^{21}$ The expression of hax1 in hematopoietic cells was also confirmed by double fluorescent in situ hybridization analysis, revealing co-localization of hax1 and cmyb, a marker of hematopoietic stem cells (Figure 1D). Notably, hax1 transcript was also detected in the brain at $4 \mathrm{dpf}$ (Figure 1G, arrow).

\section{Loss-of-function of Hax1 leads to reduced neutrophil numbers in zebrafish embryos}

To determine whether hax 1 has a role in zebrafish hematopoiesis, a morpholino (MO)-mediated gene knockdown strategy was used. Specifically, three MO targeting the hax1 gene were used (Online Supplementary Figure $S 2 A)$. One $\mathrm{MO}$ was designed against the ATG start codon site (hereafter named atg-MO), whereas the other two blocked the correct splicing of either exon 1 (hereafter named e1-MO) or exon 2 (hereafter named e2-MO), leading to a truncated Hax1 protein (Online Supplementary Figure $S 2 B-E)$. Quantitative analysis showed that wildtype hax 1 transcript levels decreased to $90 \%$ and $70 \%$ in the embryos injected with e1-MO and e2-MO, respectively (Figure 2A, Online Supplementary Figure S2F and G). Notably, injection of $\mathrm{MO}$ did not result in embryonic mal- 
A

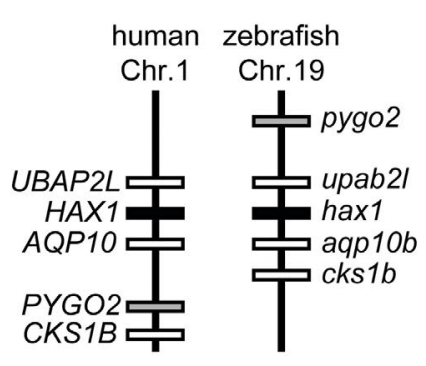

E

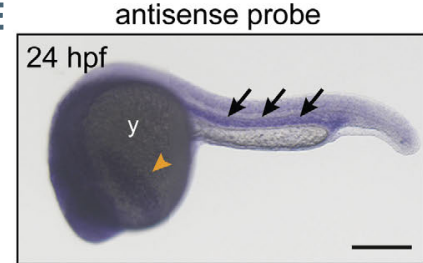

B

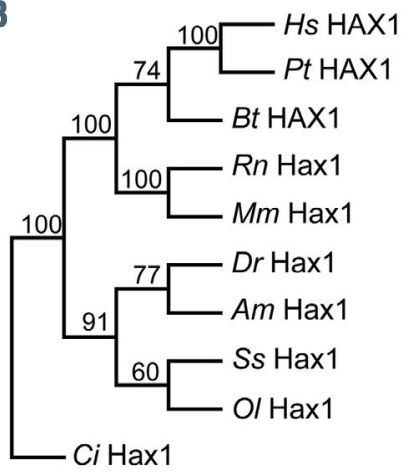

sense probe

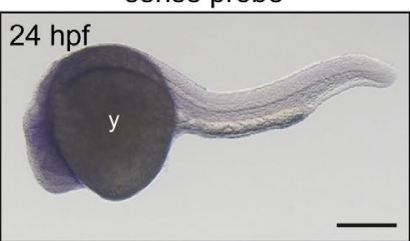

C
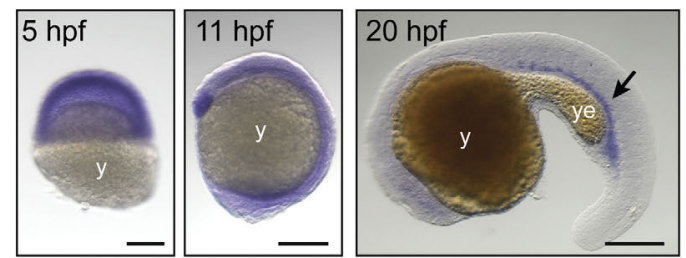

D

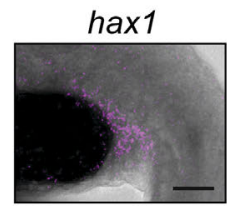

F

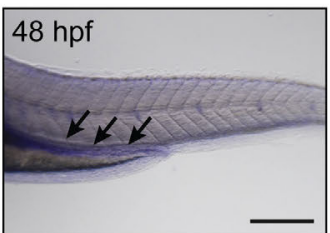

cmyb

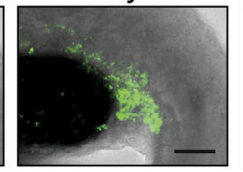

merge

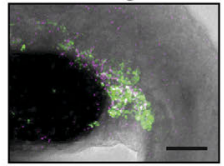

G

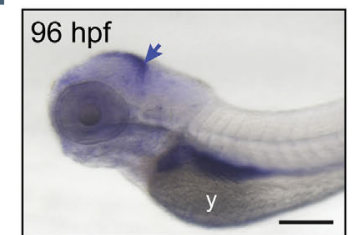

Figure 1. Characterization of zebrafish hax1. (A) Schematic comparison showing syntenic conservation of the hax1 loci in humans and zebrafish. (B) A neighbor-joining phylogenetic tree of Hax1 proteins, which was performed with 1,000 bootstrap replications. Am, Astyanax mexicanus; Bt, Bos taurus; Ci, Ciona intestinalis; Dr, Danio rerio; Hs, Homo sapiens; Mm, Mus musculus; Ol, Oryzias latipes; Pt, Pan troglodytes; Rn, Rattus norvegicus; Ss, Salmo salar. (C) Spatial hax1 expression by whole mount in situ hybridization analysis from 5 to 20 hours post-fertilization (hpf). (D) Confocal image of double fluorescent in situ hybridization of hax1 (magenta) and cmyb (green) at 20 hpf. (E-G) Spatial hax1 expression at 24 (E), 48 (F) and 96 (G) hpf. Arrows in C, E, and F indicate hax1 expression in the hematopoietic site. A sense probe was used as a negative control (E, right panel). Note that the images shown in $E$ are two images stitched together. y: yolk; ye: yolk extension. Scale bars: $100 \mu \mathrm{m}(\mathrm{C}, \mathrm{E}-\mathrm{G}), 50 \mu \mathrm{m}$ (D).

formation (Online Supplementary Figure S3). To determine whether hax1 knockdown impairs neutrophil development, two different approaches were used. First, cells expressing the neutrophil-specific marker myeloid peroxidase (mpo) were stained in $\mathrm{MO}$-injected embryos (hereafter called morphants) using WISH. Compared to wildtype embryos, the number of $m p o^{+}$cells was significantly reduced in all three morphants (Figure 2B). As a second approach, $\mathrm{MO}$ were injected separately into the zebrafish transgenic $\operatorname{tg}(m p o: g f p)$ embryos in which GFP is expressed under the control of mpo promoter. ${ }^{30}$ Consistent with WISH data, in all three morphants, the numbers of $\mathrm{GFP}^{+}$ cells were reduced in comparison to the numbers in uninjected siblings (Figure 2C) and no significant difference was observed when a control $\mathrm{MO}$ was injected (Online Supplementary Figure S4). To test the specificity of the $\mathrm{MO}$, full-length mRNA of zebrafish hax 1 was co-injected with either e1-MO or e2-MO, showing that overexpression of hax1 rescued the reduced neutrophil numbers in both morphants (Figure 2D). In addition to $\mathrm{MO}$-mediated gene knockdown, transient CRISPR-Cas9 targeting of the hax1 gene in the $\operatorname{tg}(m p o: g f p)$ line was performed. Compared with the non-injected siblings, hax 1 crispants showed significantly fewer $\mathrm{GFP}^{+}$cells in the trunk region at $2 \mathrm{dpf}$ (Online Supplementary Figure S5). Collectively, our findings suggest that hax1 has a role in zebrafish neutrophil development.

\section{Normal phagocytic activity and migratory behavior of neutrophils in hax1 morphants}

Given that Hax1 is able to interact with proteins associated with cytoskeleton machinery and is involved in the migration of cancer cells in vitro, ${ }^{38}$ we tested to what extent Hax1 loss-of-function impairs the migratory behavior of neutrophils in vivo. One way to induce an inflammatory response and chemotaxis is to inject bacteria into the notochord of zebrafish embryos. ${ }^{35} \mathrm{We}$, therefore, injected Alexa 594-conjugated Staphylococcus aureus debris into this region (Figure 3A) and then embryos were analyzed using confocal microscopy. We found a similar accumulation of neutrophils in the infected site between wild-type (Figure 3B, top panels) and hax1 morphants (Figure 3B, bottom panels). Time-lapse in vivo imaging revealed that hax 1 knockdown did not substantially affect the migration or phagocytic activity of neutrophils (Figure 3C).

\section{Normal erythropoiesis and monopoiesis in hax1 morphants}

Based on single-cell RNA sequencing databases of zebrafish hematopoietic cells, ${ }^{36,37}$ hax 1 is also expressed in erythrocytes and macrophages. We, therefore, performed tests to determine whether loss-of-function of Hax1 could affect erythropoiesis or the development of macrophages. WISH analysis showed that there were no detectable differences in the expression patterns of gata1 and hemoglobin alpha embryonic 1.1 (hbae1.1) in the morphants when compared to wild-type embryos at $1 \mathrm{dpf}$ (Figure 4A and B). These results and the presence of circulating red blood cells in the morphants (data not shown) suggest that hax1 is dispensable for embryonic erythropoiesis.

We next examined to what extent the development of myeloid cells was affected in hax 1 morphants. We tested the expression of pu.1, a regulator of monocytic differentiation. ${ }^{39}$ In $2 \mathrm{dpf}$ hax $1 \mathrm{MO}$-injected embryos, the number of pu. $1^{+}$cells appeared comparable with that in the wildtype fish (Figure 4C), which mimics data from $\mathrm{CN}$ patients. ${ }^{40}$ Analysis with markers of mature myeloid cells demonstrated a substantial decrease in l-plastin ${ }^{+}$leukocytes (Figure 4D), lyz neutrophils (Figure 4E) and g-csfr $\mathrm{r}^{+}$ 
cells (Figure 4F). The last gene is expressed in monocytes and neutrophils. In contrast, mpeg $1.1^{+}$macrophages ${ }^{41}$ were not affected (Figure 4G). With regard to definitive hematopoiesis, no difference was observed in the expression of the hematopoietic stem cell marker cmyb between wild-type and hax1 morphants at $2 \mathrm{dpf}$ (Figure 4H). Together, these results indicate that haxt is dispensable for embryonic erythropoiesis and monopoiesis.

\section{Interference with Hax1 function enhances apoptosis in embryos but not in neutrophils}

We next sought to determine the underlying molecular basis for the role of Hax1 in neutrophil development. To date, two main observations associated with HAX1 deficiency in $\mathrm{CN}$ patients are increased apoptosis of myeloid progenitors, ${ }^{6}$ and decreased activity of the G-CSF signal transduction pathway. ${ }^{18}$ Based on these findings, we examined to what extent cellular viability and the G-CSF signaling pathway were affected in hax1 morphants. We used three approaches to identify apoptotic cells. First, a terminal deoxynucleotidyl transferase dUTP nick end labeling (TUNEL) assay was performed to detect apoptotic cells. Compared to their wild-type counterparts, hax1 morphants exhibited increased apoptosis at $1 \mathrm{dpf}$ (Figure $5 \mathrm{~A}$ and $\mathrm{B}$ ). The increased apoptosis at $1 \mathrm{dpf}$ was specific to the hax1 knockdown fish because no significant differ- ence was observed when a control $\mathrm{MO}$ was injected (Figure 5B). By $2 \mathrm{dpf}$, the number of apoptotic cells was comparable between morphants and uninjected embryos (Figure 5C, Online Supplementary Figure S6). As a second approach, we stained wild-type embryos and hax 1 morphants with the fluorescent dye acridine orange (Figure 5D). Similar to the TUNEL assay, this showed that the number of apoptotic cells was increased in the hax1 morphants at $1 \mathrm{dpf}$ (Figure 5E), but not at $2 \mathrm{dpf}$ (Figure 5F). Strikingly, apoptotic cells stained with acridine orange as well as TUNEL were scattered throughout the embryo and not preferentially associated with sites of hematopoiesis. In support of this notion, we incubated wild-type and hax1-injected $\operatorname{tg}(1 y z: d s R E D)$ embryos with a caspase 3/7 reporter, which produces a green fluorogenic response upon cleavage by activated caspase- 3 or caspase-7. Confocal imaging of $2 \mathrm{dpf}$ transgenic $\operatorname{tg}(l y z: d s R E D)$ embryos (Figure $5 \mathrm{G}$ ) showed that the frequency of dsRED ${ }^{+}$cells stained with the caspase 3/7 reporter was comparable in the wild-type and hax1 morphants at $2 \mathrm{dpf}$ (Figure 5H). It is also worth noting that interference with Hax1 function did not affect cell proliferation in embryos (Online Supplementary Figure S7). These findings, therefore, indicate that hax 1 knockdown enhances apoptosis in zebrafish embryos at early stages, but not in neutrophils.
A
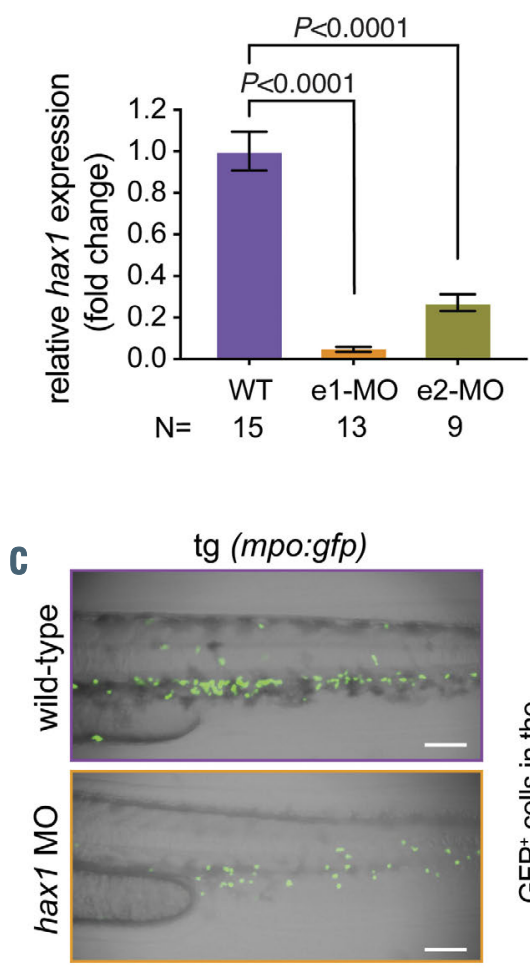

B

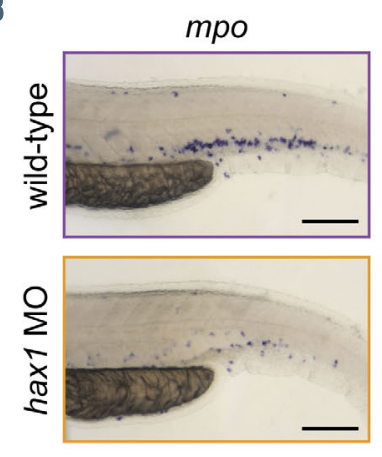

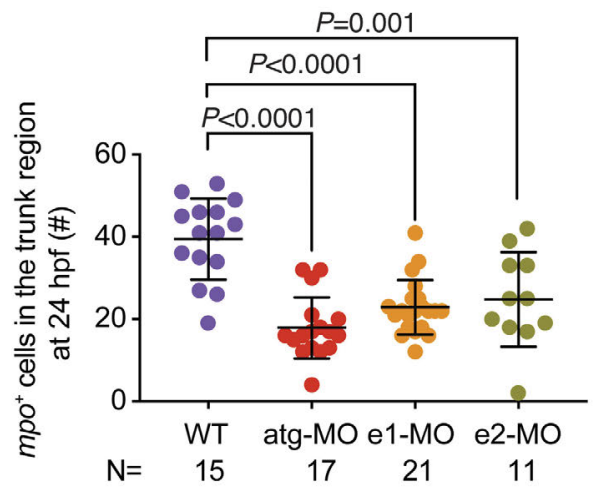

D

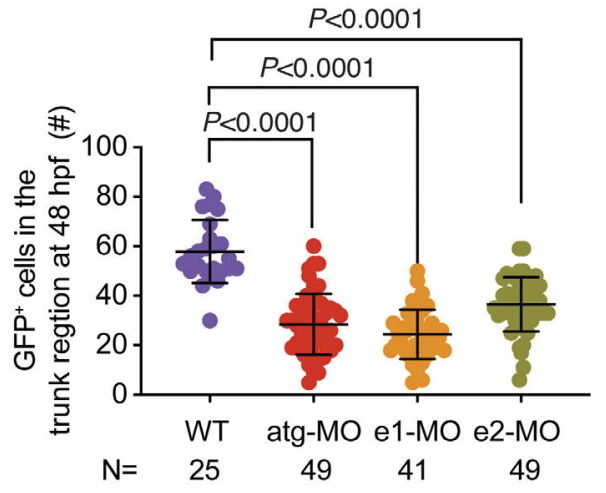

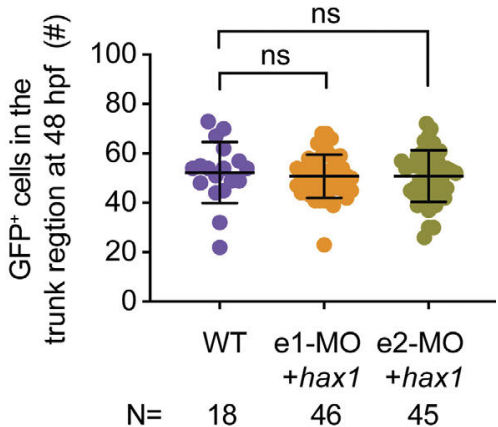

Figure 2. Knockdown of hax1 impairs neutrophil development. (A) Relative change of wild-type hax1 transcript in the hax1 morphants compared with wild-type (WT) using quantitative polymerase chain reaction. $\mathrm{N}$ indicates number of biological replicates. (B) Representative images of $m p o-s t a i n e d$ cells in WT and e1-MO injected embryos (left panel). Note that each stained cell represents a neutrophil. The right panel shows numbers of mpo stained cells in the trunk region at 24 hours postfertilization (hpf). (C) Injection of hax1 morpholinos (MO) in the tg(mpo:gfp) line. The left panel shows representative images of uninjected (WT) and hax1 e1-M0 injected transgenic embryos at $48 \mathrm{hpf}$. The right panel shows numbers of green fluorescent protein-positive cells in the trunk region. (D) Co-injection of e1-MO or e2MO morpholinos with hax1 mRNA rescued the reduced neutrophil numbers in the tg(mpo:gfp) line. Scale bars indicate $100 \mu \mathrm{m}$. Each dot represents an individual embryo. Data are means \pm standard deviation. 
Granulocyte colony-stimulating factor rescues reduced neutrophil numbers in the hax1 morphants

Next, two approaches were used to evaluate the expression of hcls 1 , cebpa and cebpb, which are activated by G-CSF. First, total RNA was isolated from morphants and uninjected embryos at $2 \mathrm{dpf}$. Quantitative reverse transcriptase polymerase chain reaction analysis was performed to determine the levels of expression of hist, cebpa and cebpb. This revealed a downregulation of the expression levels of $h c l s 1$ and cebpa, while the expression of cebpl was upregulated in the haxt morphants (Figure 6A). As a second approach, cells expressing cebpa or cebpb were stained using WISH. Similarly, the number of cebpa cells was reduced (Figure 6B), while the number of cebpb cells was increased (Figure 6C) in the hax1 morphants. These findings raised the question of whether overexpression of $g$-csf is sufficient to rescue the reduced number of neutrophils in hax 1 morphants. To address this question, a heat-inducible promoter ${ }^{34}$ was used to ectopically express zebrafish g-csfa at $1 \mathrm{dpf}$ (Figure 6D).
A

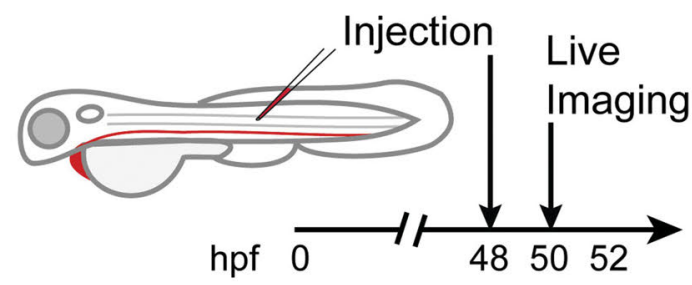

B

GFP
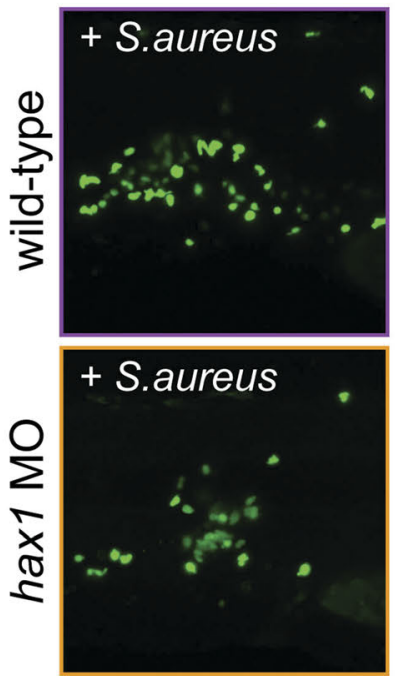

C

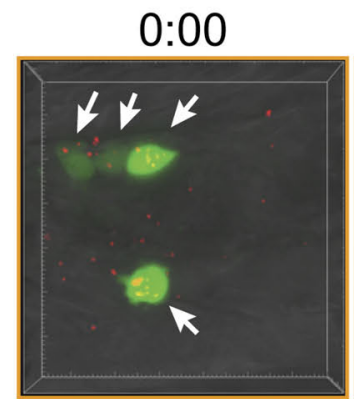

$3: 50$

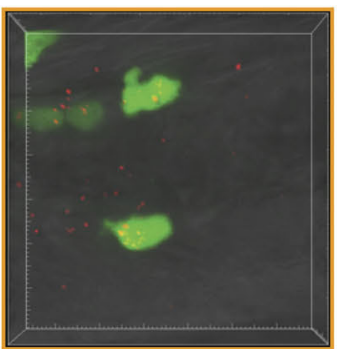

Alexa 594
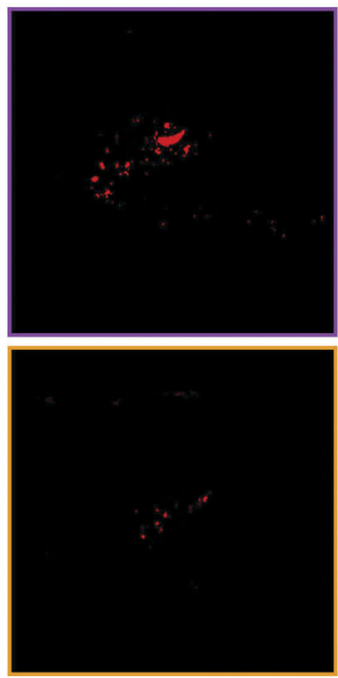

$1: 17$

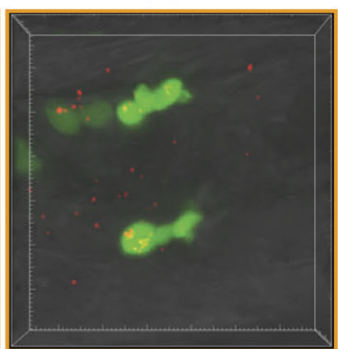

5:06

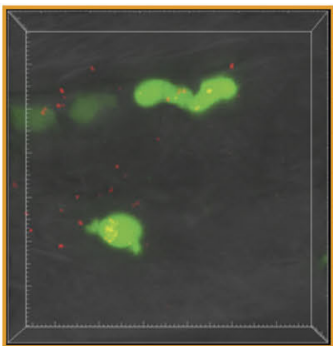

Merge+DIC
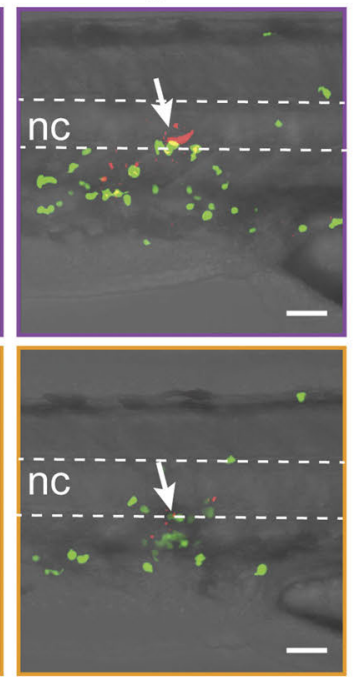

$2: 33$

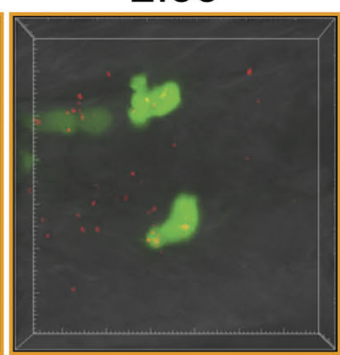

$6: 22$

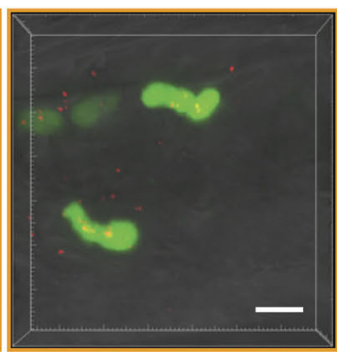

Figure 3. Migration and phagocytosis of neutrophils in the hax1 morphants. (A) Experimental design. (B) Injection of Alexa-594 conjugated Staphylococcus aureus debris into the notochord of $\operatorname{tg}(m p o: g f p)$ embryos at 2 days post-fertilization (dpf). Arrows indicate the injected site. Dashed lines indicate the position of the notochord. (C) Still photographs from a time-lapse recording illustrating the migration and phagocytic activity of neutrophils (arrows) in the hax 1 morphants. Numbers indicate time in minutes. Scale bars, $40 \mu \mathrm{m}$ (B) and $10 \mu \mathrm{m}$ (C). GFP: green fluorescent protein; nc: notochord. 
Consistent with a previous study, ${ }^{22}$ an average 1.7-fold increase of $\mathrm{mpo}^{+}$cells was observed when g-csfa was ectopically expressed in wild-type embryos (Figure 6E). Next, the g-csfa inducible construct was co-injected with hax1 e1-MO into embryos. Heat shock was performed at
$1 \mathrm{dpf}$ and $\mathrm{GFP}^{+}$embryos were selected for WISH analysis As expected, embryos injected with e1-MO displayed reduced neutrophil numbers. However, when g-csfa was induced in the morphants, we observed that the number of neutrophils was increased to a level which was compa-
A

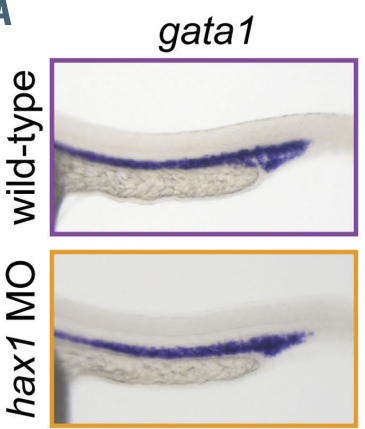

C

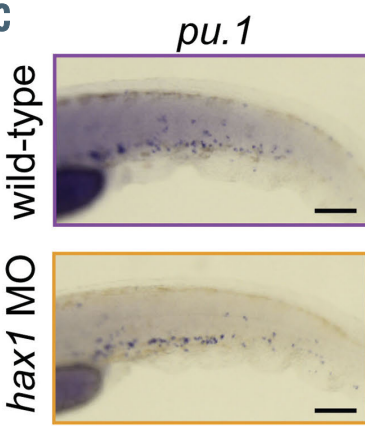

E
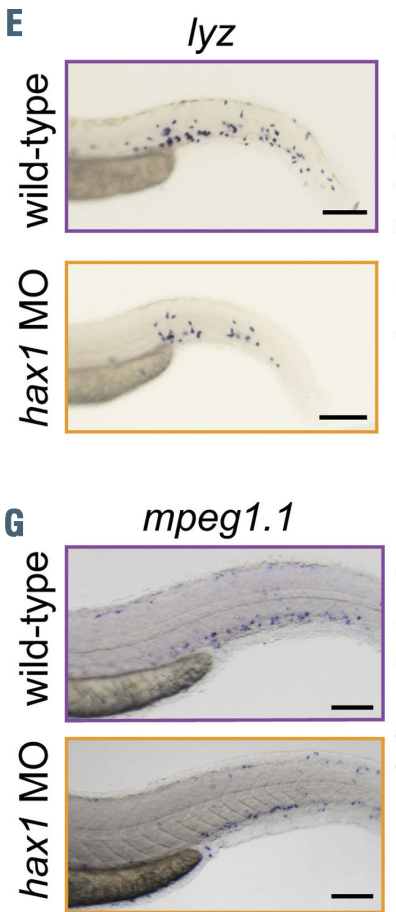
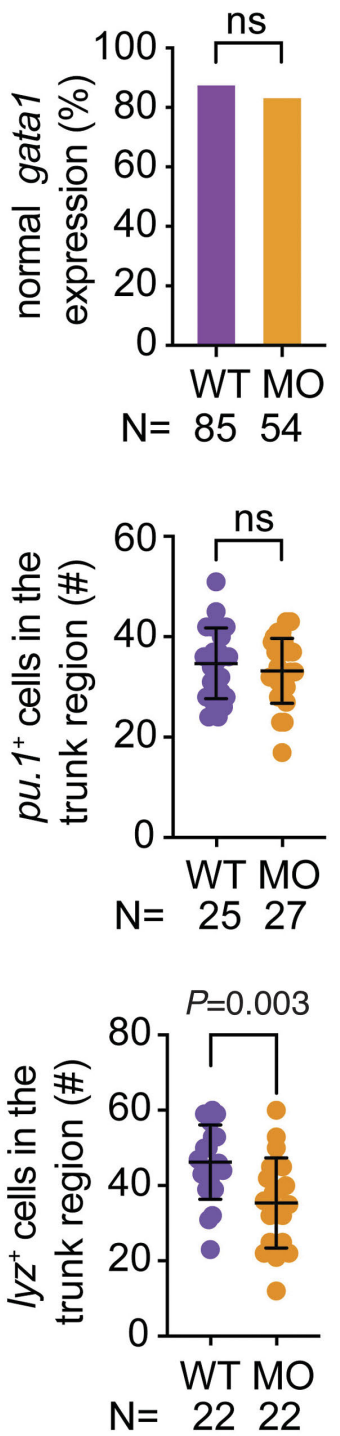

$\mathrm{N}=2527$

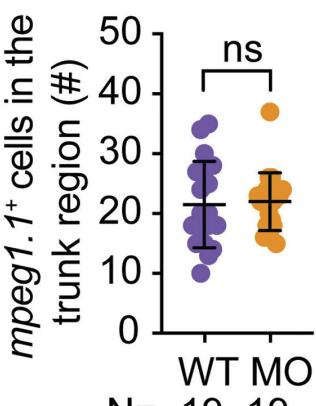

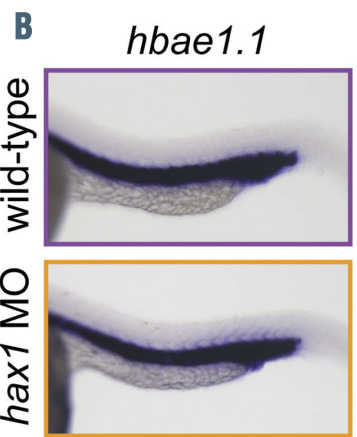
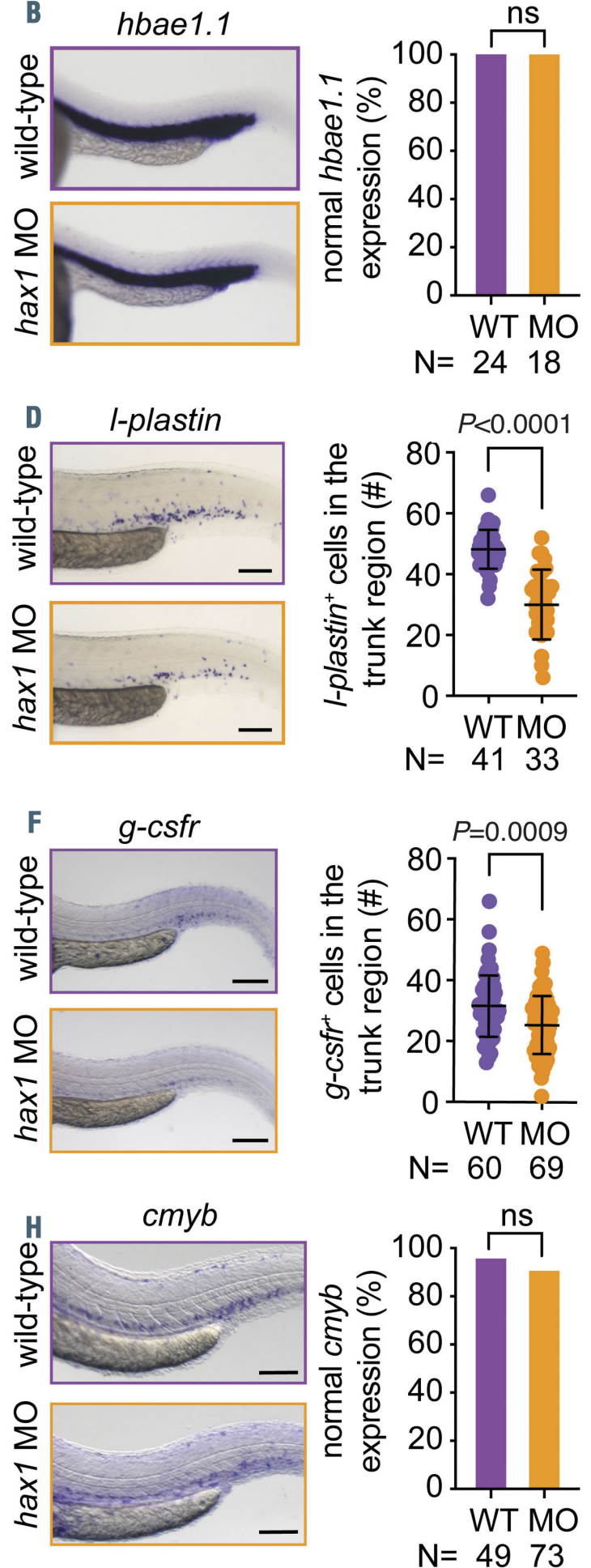

Figure 4. Normal development of erythrocytes, macrophages and hematopoietic stem cells in the hax1 morphants. (A-H) Left panels show representative images of whole mount in situ hybridization for gata1 (A), hbae1.1 (B), pu.1 (C), l-plastin (D), lyz (E), g-csfr (F), mpeg1.1 (G) and cmyb (H) expression in uninjected (WT) and hax1 e1-MO injected embryos at 24 hours post-fertilization (hpf) $(\mathrm{A}, \mathrm{B})$ or $48 \mathrm{hpf}(\mathrm{C}-\mathrm{H})$. Right panels show quantitative numbers of stained cells in the trunk region. Scale bars indicate $100 \mu \mathrm{m}$. Each dot in C-G represents an individual embryo. Data are means \pm standard deviation. 
rable with that in the control group (Figure 6E), indicating that $g$-csfa is sufficient to reverse the reduced neutrophil numbers in hax 1 morphants.

\section{Discussion}

In this study, we show that hax1 is indispensable for zebrafish granulopoiesis. This is the first study demonstrating that hax1 is required for neutrophil development in a vertebrate other than humans. Our data revealed that knockdown of hax 1 reduces the expression of cebpa and hcls1 genes, two downstream target genes of the G-csf signaling pathway. This result is in agreement with our previous in vitro study, in which knockdown of HAX1 in $\mathrm{CD} 34^{+}$progenitor cells impaired granulocytic differentiation by reducing the levels of CEBPA and HCLS1 expression. ${ }^{18}$ Similarly, CEBPA expression is severely diminished in $\mathrm{CN}$ patients with $H A X 1$ deficiency. $\mathrm{C} / \mathrm{EBP} \alpha$ is a transcription factor involved in steady-state granulopoiesis, and regulates G-CSFR expression through a positive feed- back loop. ${ }^{25}$ This regulatory relationship between $\mathrm{C} / \mathrm{EBP} \alpha$ and G-CSFR appears to be evolutionarily conserved between zebrafish and humans because zebrafish cebpa mutants also display reduced $g$-csfr expression. ${ }^{42}$ Although we did not provide a direct assessment of G-CSF signaling in zebrafish hax1-deficient neutrophils, the reduced levels of expression of hcls1 and cebpa, two direct target genes of G-CSF signaling, together with decreased $g$-csfr ${ }^{+}$cells in the morphants support the notion that hax 1 has a role in G-CSF signaling, which is in agreement with previous in vitro data. ${ }^{18}$ It is, however, important to stress that our data do not rule out the possibility that hax 1 knockdown reduced the number of cebpa-expressing cells rather than decreasing the cebpa expression level in each myeloid cell. Nevertheless, the expression of cebpb was increased in the hax 1 morphants. C/EBP $\beta$ functions as the main transcriptional regulator for emergency granulopoiesis, ${ }^{43}$ and $\mathrm{CN}$ patients with $H A X 1$ deficiency also show an elevated level of CEBPB expression. . $^{18,44}$

Previous studies have shown that HAX1 has an antiapoptotic function and interacts with a variety of intracel-
A

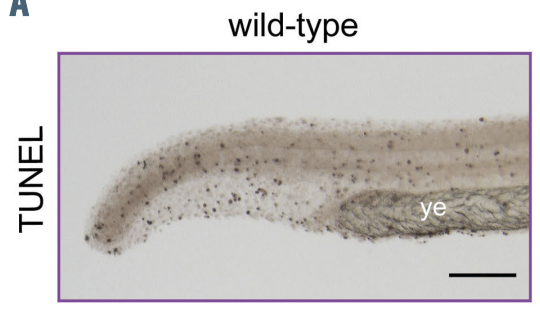

D

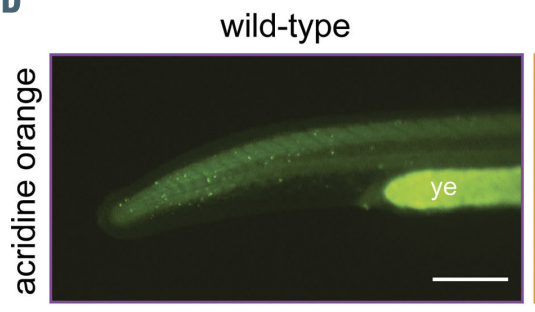

hax1 MO

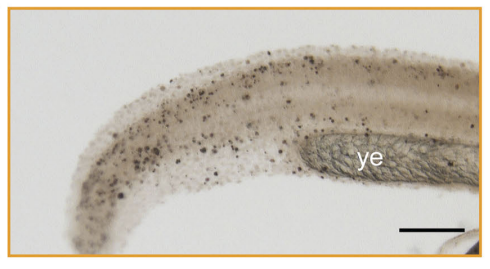

hax1 MO

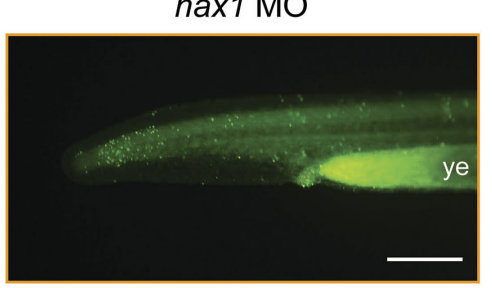

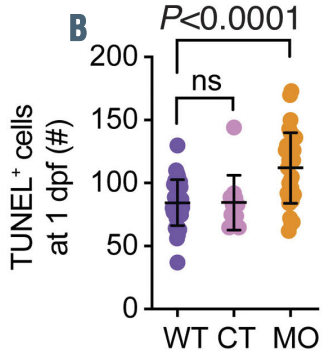

$\mathrm{N}=\begin{array}{lll}35 & 11 & 35\end{array}$

E

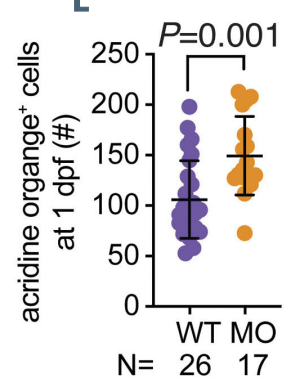

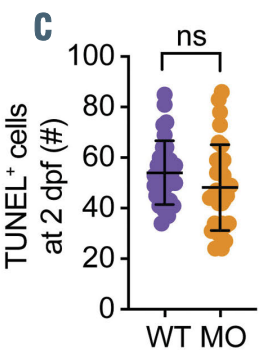

$\mathrm{N}=2135$

F

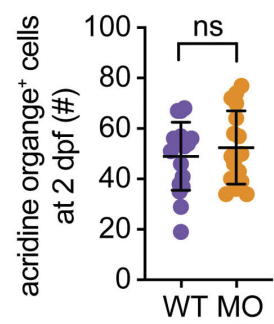

$N=1817$
G

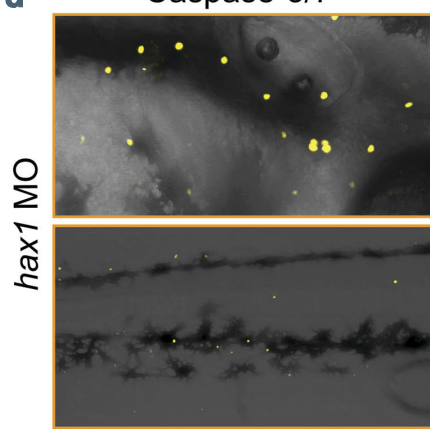

$\operatorname{tg}(l y z: d s R E D)$
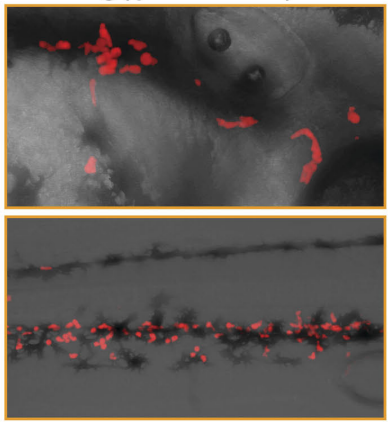

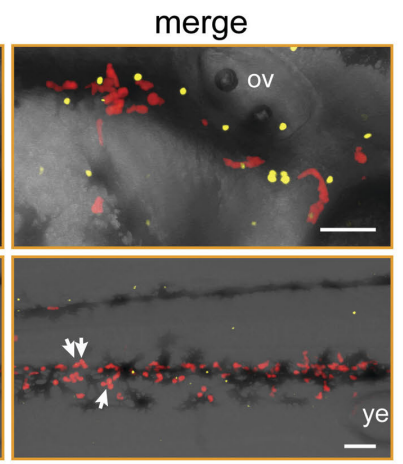

H

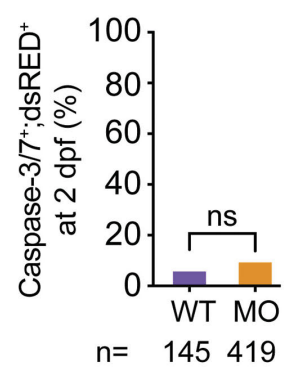

Figure 5. Enhanced apoptosis by hax1 knockdown. (A) Representative images of TUNEL-positive cells in wild-type (WT) and hax1 morphants (MO) at 1 day post-fertilization (dpf). (B, C) Quantitative numbers of TUNEL-positive cells in the trunk region at $1 \mathrm{dpf}(\mathrm{B})$ and $2 \mathrm{dpf}(\mathrm{C})$. Note injection of control (CT) morpholino did not significantly increase the number of TUNEL-positive cells. (D) Representative images of acridine orange-stained cells in WT and hax1 MO at $1 \mathrm{dpf}$. (E, F) Quantitative numbers of acridine orange-stained cells in the trunk region at $1 \mathrm{dpf}(\mathrm{E})$ and $2 \mathrm{dpf}(\mathrm{F})$. (G) Representative images from the head (top panel) and trunk region (bottom panel) of the $\operatorname{tg}(I y z: d s R E D)$ embryos injected with hax1 MO showing cells stained with caspase-3/7 reporter (yellow) and neutrophils (red) at 2 dpf. (H) Frequency of caspase-3/7 and dsRED double positive cells in WT and hax1 morphants. $n$ indicates number of dsRED ${ }^{+}$cells counted from three wild-type embryos (WT) and 10 morphants (MO) at $2 \mathrm{dpf}$. Each dot in B, C, E and F represents an individual embryo. Data are means \pm standard deviation. Scale bars: $100 \mu \mathrm{m}$ (A, D) and $50 \mu \mathrm{m}$ (G). ov: otic vesicle; ye: yolk extension. 
A

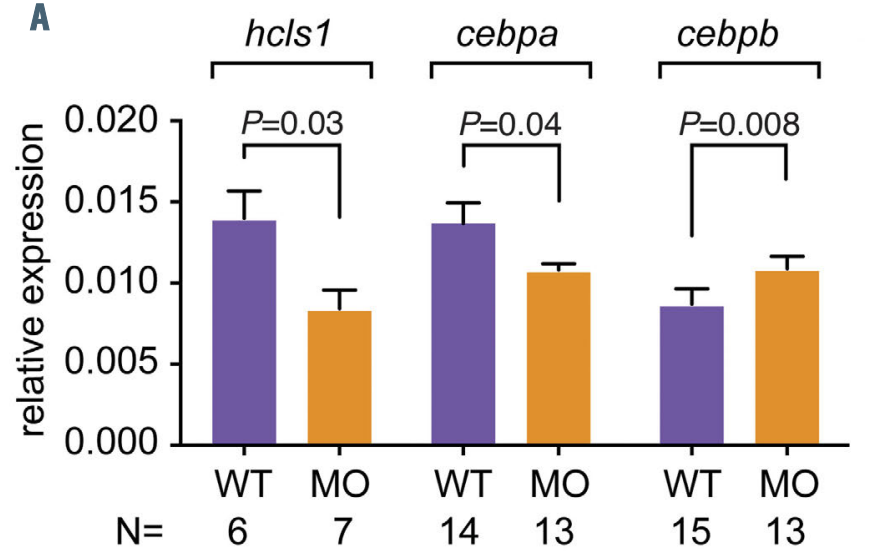

D
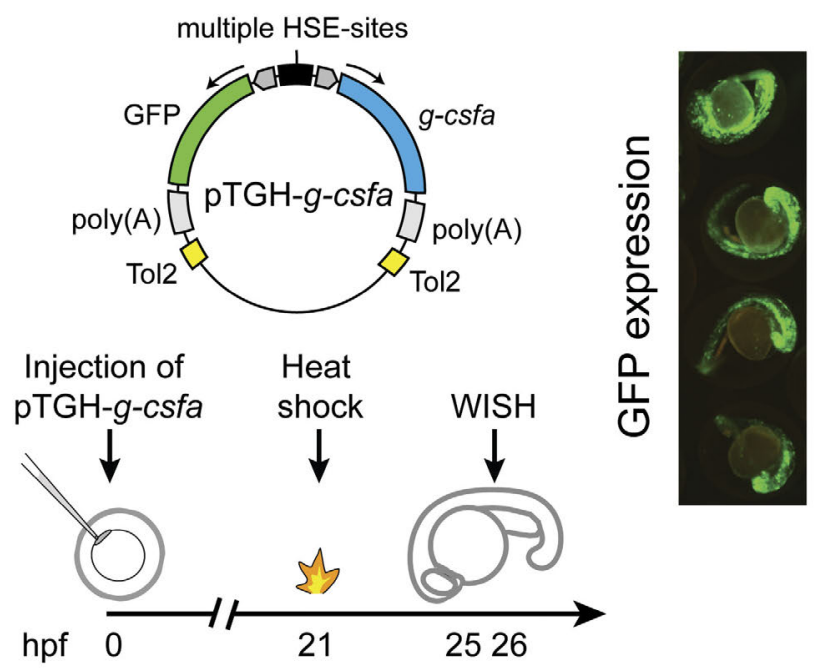

B

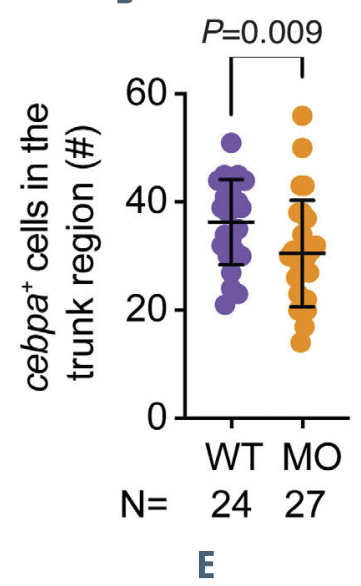

C

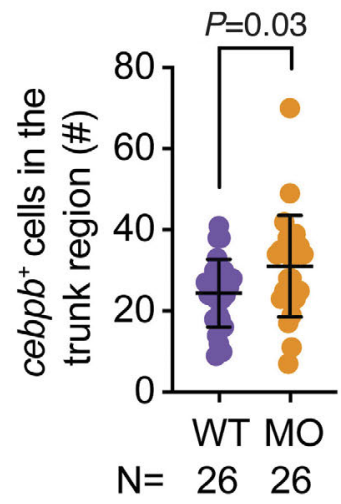

\section{ns}

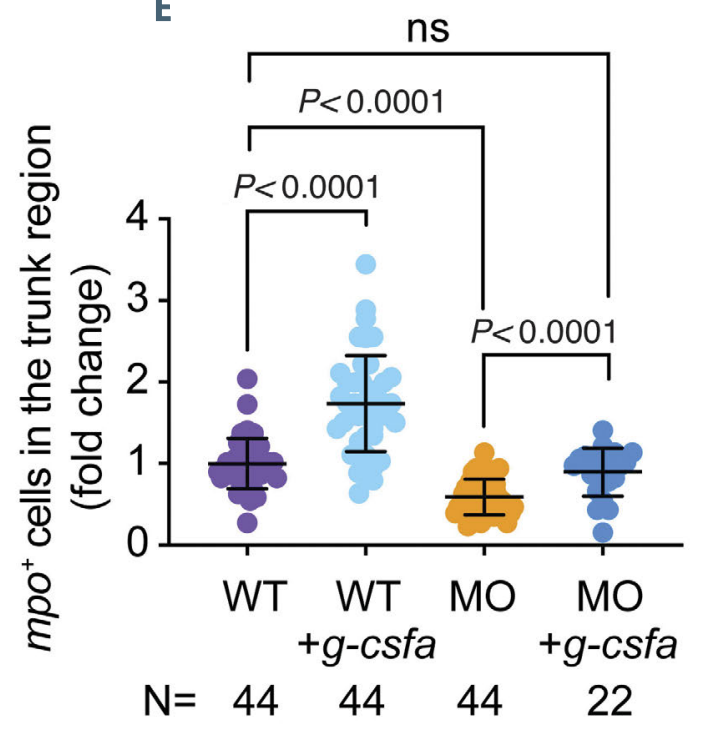

Figure 6. G-csfa induction rescued the reduced neutrophil numbers in the hax1 morphants. (A) Relative expression of hcls1, cebpa, cebpb in wild-type (WT) and morphants (MO) at 2 days post-fertilization (dpf). The $\beta$-actin gene was used as an internal control for normalization. $\mathrm{N}$ indicates number of biological replicates. (B, C) Quantitative numbers of cebpa- and cebpb-expressing cells in the trunk region of WT and MO at 2 dpf. (D) The top panel illustrates the bi-directional construct (pTGH-g-csfa) used to ectopically induce the zebrafish g-csfa cDNA. Note that green fluorescent protein (GFP) expression was used as a positive control for induction (4 representative embryos are shown in the right panel). The lower panel outlines the timing of the experiment. (E) Fold change of $m p o^{+}$cells in the trunk region of embryos at 25 hours post-fertilization (hpf). Each dot represents an individual embryo. N indicates number of embryos. Data are means \pm standard deviation.

lular apoptosis-related proteins. ${ }^{15,45}$ Consequently, Hax1deficient mice display neuronal apoptosis. ${ }^{15}$ Increased apoptosis was also observed in the bone marrow myeloid progenitor cells of $\mathrm{CN}$ patients with a HAX1 mutation. ${ }^{46}$ Knockdown of zebrafish hax 1 increased apoptosis at $1 \mathrm{dpf}$, as determined by a TUNEL assay and acridine orange staining. However, cell death was not associated with the hematopoietic tissue. Furthermore, knockdown of hax1 did not enhance apoptosis in neutrophils. Hence, zebrafish Hax1 has an anti-apoptotic role during early embryonic development, but increased cell death is most likely not the main reason for the reduced neutrophil numbers. These results contradict previous data indicating that autosomal recessive mutations in the human HAX1 gene are associated with increased apoptosis in myeloid cells. ${ }^{6}$

In human, two alternatively spliced isoforms of HAX1 have been identified. ${ }^{4}$ Patients with HAX1 mutations affecting the full-length transcript or the splice variant I develop $\mathrm{CN}$, whereas patients with mutations affecting the splice isoforms I and II develop CN with neurological abnormalities. ${ }^{5,10}$ The isoform II uses an alternate in-frame splice site producing a shortened exon 2 , and is expressed in the brain but not in the bone marrow. ${ }^{10}$ In zebrafish, a sole hax 1 transcript was identified by amplification from the embryonic cDNA. Interestingly, hax1 transcript was also detected in a brain region where newborn neurons are continuously added and contribute to sensory information processing, akin to the superior colliculus in mammals. ${ }^{47}$ Nevertheless, the role of Hax1 in zebrafish neurogenesis remains to be elucidated.

Collectively, our data have established zebrafish as an in vivo model for HAX1-associated neutropenia, filling the gap between in vitro models and clinical assessment. Given that the zebrafish is a valuable model for studying driver mutations underlying disease pathogenesis in acute myeloid leukemia and myelodysplastic syndromes, ${ }^{48}$ a stable hax1-deficient zebrafish line will help investigations into the leukemogenic role of Hax1, alone and in combination with other gene mutations. ${ }^{49}$ Besides being an important cancer model, the zebrafish also represents a reliable platform to perform screens of large compounds and assess their therapeutic relevance. ${ }^{50}$ Therefore, we 
anticipate that our model will serve as a platform to identify new avenues for developing tailored therapeutic strategies for patients with $\mathrm{CN}$.

\section{Disclosures}

No conflicts of interest to disclose.

\section{Contributions}

$L D$ and $B B$ made initial observations; $L D$ performed most of the experiments and contributed to the design of the work; NA performed experiments in the transgenic lines; $A M D$ performed quantitative polymerase chain reaction, immunostaining and fluorescence in situ hybridization analysis; $K W$ and JS provided useful insights and interpreted the data. BB supervised and supported the study and wrote the manuscript. All authors read and edited the manuscript.

\section{Acknowledgments}

The authors would like to thank Patrick Müller (Fredrich Miescher Laboratory, Tübingen) for providing the wild-type TE strain of zebrafish and transgenic lines, Jochen Wittbrodt (Center for Organismal Studies, Heidelberg University) for providing the Cas9 construct, Annisa Claasen and Christine Gottschalk for technical help, and the Institute of Medical Virology and Microbiology for continuous support in confocal microscopy.

\section{Funding}

The work in $B B^{\prime}$ s laboratory is supported by the Deutsche Forschungsgemeinschaft (BA 5766/3-1), Deutsche José Carreras Leukämie-Stiftung (DJCLS11 R/2018), and Wilhelm-SanderStiftung.

\section{References}

1. Skokowa J, Dale DC, Touw IP, Zeidler C, Welte K. Severe congenital neutropenias. Nat Rev Dis Primers. 2017;3:17032.

2. Klein C. Kostmann's disease and HCLS1associated protein X-1 (HAX1). J Clin Immunol. 2017;37(2):117-122.

3. Boztug K, Ding XQ, Hartmann $\mathrm{H}$, et al. HAX1 mutations causing severe congenital neuropenia and neurological disease lead to cerebral microstructural abnormalities documented by quantitative MRI. Am J Med Genet A. 2010;152A(12):3157-3163.

4. Fadeel B, Grzybowska E. HAX-1: a multifunctional protein with emerging roles in human disease. Biochim Biophys Acta. 2009;1790(10):1139-1148.

5. Germeshausen M, Grudzien M, Zeidler C, et al. Novel HAX1 mutations in patients with severe congenital neutropenia reveal isoform-dependent genotype-phenotype associations. Blood. 2008;111(10):49544957.

6. Klein C, Grudzien M, Appaswamy G, et al. HAX1 deficiency causes autosomal recessive severe congenital neutropenia (Kostmann disease). Nat Genet. 2007; 39(1):86-92.

7. Yetgin S, Olcay L, Koc A, Germeshausen M. Transformation of severe congenital neutropenia to early acute lymphoblastic leukemia in a patient with HAX1 mutation and without G-CSF administration or receptor mutation. Leukemia. 2008; 22(9):1797.

8.Zeidler C, Germeshausen M, Klein C, Welte K. Clinical implications of ELA2-, HAX1-, and G-CSF-receptor (CSF3R) mutations in severe congenital neutropenia. Br J Haematol. 2009:144(4):459-467.

9. Rosenberg PS, Zeidler C, Bolyard AA, et al. Stable long-term risk of leukaemia in patients with severe congenital neutropenia maintained on G-CSF therapy. Br J Haematol. 2010;150(2):196-199.

10. Carlsson G, van't Hooft I, Melin M, et al. Central nervous system involvement in severe congenital neutropenia: neurological and neuropsychological abnormalities associated with specific HAX1 mutations. J Intern Med. 2008;264(4):388-400.

11. Hippe A, Bylaite $M$, Chen $M$, et al. Expression and tissue distribution of mouse Hax1. Gene. 2006;379:116-126.

12. Grzybowska EA, Zayat V, Konopinski R, et al. HAX-1 is a nucleocytoplasmic shuttling protein with a possible role in mRNA pro- cessing. FEBS J. 2013;280(1):256-272.

13. Suzuki Y, Demoliere C, Kitamura D, Takeshita $\mathrm{H}$, Deuschle U, Watanabe T. HAX-1, a novel intracellular protein, localized on mitochondria, directly associates with HS1, a substrate of Src family tyrosine kinases. J Immunol. 1997;158(6):2736-2744.

14. Yap SV, Vafiadaki E, Strong J, KontrogianniKonstantopoulos A. HAX-1: a multifaceted antiapoptotic protein localizing in the mitochondria and the sarcoplasmic reticulum of striated muscle cells. J Mol Cell Cardiol. 2010;48(6):1266-1279.

15. Chao JR, Parganas E, Boyd K, Hong CY, Opferman JT, Ihle JN. Hax1-mediated processing of HtrA2 by Parl allows survival of lymphocytes and neurons. Nature. 2008:452(7183):98-102.

16. Gallagher AR, Cedzich A, Gretz N, Somlo $S$, Witzgall R. The polycystic kidney disease protein PKD2 interacts with Hax-1, a protein associated with the actin cytoskeleton. Proc Natl Acad Sci U S A. 2000; 97(8):4017-4022

17. Wolkerstorfer S, Schwaiger E, Rinnerthaler $M$, et al. HAX1 deletion impairs BCR internalization and leads to delayed BCR-mediated apoptosis. Cell Mol Immunol. 2016;13(4):451-461.

18. Skokowa J, Klimiankou M, Klimenkova O, et al. Interactions among HCLS1, HAX1 and LEF-1 proteins are essential for G-CSFtriggered granulopoiesis. Nat Med. 2012;18(10):1550-1559

19. Morishima T, Watanabe K, Niwa A, et al. Genetic correction of HAX1 in induced pluripotent stem cells from a patient with severe congenital neutropenia improves defective granulopoiesis. Haematologica. 2014;99(1):19-27.

20. Pittermann E, Lachmann N, MacLean G, et al. Gene correction of HAX1 reversed Kostmann disease phenotype in patientspecific induced pluripotent stem cells. Blood Adv. 2017;1(14):903-914

21. Avagyan S, Zon LI. Fish to learn: insights into blood development and blood disorders from zebrafish hematopoiesis. Hum Gene Ther. 2016;27(4):287-294.

22. Stachura DL, Svoboda O, Campbell CA, et al. The zebrafish granulocyte colony-stimulating factors (Gcsfs): 2 paralogous cytokines and their roles in hematopoietic development and maintenance. Blood. 2013;122(24):3918-3928.

23. Pazhakh V, Clark S, Keightley MC, Lieschke GJ. A GCSFR/CSF3R zebrafish mutant models the persistent basal neu- trophil deficiency of severe congenital neutropenia. Sci Rep. 2017;7:44455.

24. Basheer F, Rasighaemi P, Liongue C, Ward AC. Zebrafish granulocyte colony-stimulating factor receptor maintains neutrophil number and function throughout the life span. Infect Immun. 2019;87(2):e00793-18.

25. Liongue C, Hall CJ, O'Connell BA, Crosier P, Ward AC. Zebrafish granulocyte colonystimulating factor receptor signaling promotes myelopoiesis and myeloid cell migration. Blood. 2009;113(11):2535-2546.

26. Howe K, Clark MD, Torroja CF, et al. The zebrafish reference genome sequence and its relationship to the human genome. Nature. 2013;496(7446):498-503.

27. Oyarbide U, Topczewski J, Corey SJ. Peering through zebrafish to understand inherited bone marrow failure syndromes. Haematologica. 2019;104(1):13-24.

28. Walters KB, Green IM, Surfus JC, Yoo SK Huttenlocher A. Live imaging of neutrophil motility in a zebrafish model of WHIM syndrome. Blood. 2010;116(15):2803-2811.

29. Zhang CY, Yin HM, Wang $\mathrm{H}$, et al Transforming growth factor-beta1 regulates the nascent hematopoietic stem cell niche by promoting gluconeogenesis. Leukemia. 2018;32(2):479-491.

30. Renshaw SA, Loynes CA, Trushell DM Elworthy S, Ingham PW, Whyte MK. A transgenic zebrafish model of neutrophilic inflammation. Blood. 2006;108(13):39763978

31. Hall C, Flores MV, Storm T, Crosier K, Crosier P. The zebrafish lysozyme C promoter drives myeloid-specific expression in transgenic fish. BMC Dev Biol. 2007;7:42.

32. Fink M, Flekna G, Ludwig A, Heimbucher $\mathrm{T}$, Czerny $\mathrm{T}$. Improved translation efficiency of injected mRNA during early embryonic development. Dev Dyn. 2006; 235(12):3370-3378.

33. Stemmer M, Thumberger T, Del Sol Keyer M, Wittbrodt J, Mateo JL. CCTop: an intuitive, flexible and reliable CRISPR/Cas9 target prediction tool. PLoS One. 2015 10(4):e0124633.

34. Bajoghli B, Aghaallaei N, Heimbucher T, Czerny T. An artificial promoter construct for heat-inducible misexpression during fish embryogenesis. Dev Biol. 2004; 271(2):416-430.

35. Kuri P, Ellwanger K, Kufer TA, Leptin M Bajoghli B. A high-sensitivity bi-directional reporter to monitor NF-kappaB activity in cell culture and zebrafish in real time. J Cell Sci. 2017;130(3):648-657. 
36. Tang Q, Iyer S, Lobbardi R, et al. Dissecting hematopoietic and renal cell heterogeneity in adult zebrafish at single-cell resolution using RNA sequencing. J Exp Med. 2017;214(10):2875-2887.

37. Athanasiadis EI, Botthof JG, Andres H, Ferreira L, Lio P, Cvejic A. Single-cell RNAsequencing uncovers transcriptional states and fate decisions in haematopoiesis. Nat Commun. 2017;8(1):2045.

38. Cavnar PJ, Berthier E, Beebe DJ, Huttenlocher A. Hax1 regulates neutrophil adhesion and motility through RhoA. J Cell Biol. 2011;193(3):465-473.

39. Tenen DG, Hromas R, Licht JD, Zhang DE. Transcription factors, normal myeloid development, and leukemia. Blood. 1997; 90(2):489-519.

40. Skokowa J, Welte K. Dysregulation of myeloid-specific transcription factors in congenital neutropenia. Ann N Y Acad Sci. 2009;1176:94-100
41. Ellett $F$ Pase L Hayman JW Andrianopoulos A, Lieschke GJ. mpeg1 promoter transgenes direct macrophagelineage expression in zebrafish. Blood. 2011;117(4):e49-56

42. Dai Y, Zhu L, Huang Z, et al. Cebpalpha is essential for the embryonic myeloid progenitor and neutrophil maintenance in zebrafish. J Genet Genomics. 2016; 43(10):593-600.

43. Manz MG, Boettcher S. Emergency granulopoiesis. Nat Rev Immunol. 2014; 14(5):302-314

44. Skokowa J, Welte K. Defective G-CSFR signaling pathways in congenital neutropenia. Hematol Oncol Clin North Am. 2013; 27(1):75-88, viii.

45. Yap SV, Koontz JM, KontrogianniKonstantopoulos A. HAX-1: a family of apoptotic regulators in health and disease. J Cell Physiol. 2011;226(11):2752-2761.

46. Carlsson G, Aprikyan AA, Tehranchi R, et al. Kostmann syndrome: severe congenital neutropenia associated with defective expression of $\mathrm{Bcl}-2$, constitutive mitochondrial release of cytochrome $\mathrm{c}$, and excessive apoptosis of myeloid progenitor cells. Blood. 2004;103(9):3355-3361.

47. Boulanger-Weill J, Sumbre G. Functional integration of newborn neurons in the zebrafish optic tectum. Front Cell Dev Biol. 2019;7:57.

48. Baeten JT, de Jong JLO. Genetic models of leukemia in zebrafish. Front Cell Dev Biol. 2018;6:115

49. Skokowa J, Steinemann D, KatsmanKuipers JE, et al. Cooperativity of RUNX1 and CSF3R mutations in severe congenital neutropenia: a unique pathway in myeloid leukemogenesis. Blood. 2014;123(14):22292237.

50. MacRae CA, Peterson RT. Zebrafish as tools for drug discovery. Nat Rev Drug Discov. 2015;14(10):721-731. 